\title{
Introducing the first issue of Future Medicine's new journal - Bioelectronics in Medicine
}

\author{
Robert K Shepherd*,1 \\ ${ }^{1}$ Principal Scientist, Bionics Institute, Professor of Medical Bionics, University of Melbourne, 384-388 Albert St, East Melbourne Vic \\ 3002, Australia \\ * Author for correspondence: Tel.: +61 39667 7500; Direct line: +61 39667 7597; Fax: +61 39667 7518; \\ rshepherd@bionicsinstitute.org; www.bionicsinstitute.org
}

First draft submitted: 14 November 2017; Accepted for publication: 16 November 2017; Published online: 14 December 2017

Welcome to the inaugural issue of Bioelectronics in Medicine, a brand new journal servicing the rapidly emerging new sector in neural prostheses of bioelectronics.

Neural prostheses or neural stimulators are active implantable devices that provide therapeutic intervention, sensory feedback or improved motor control via electrical stimulation of neural tissue in response to trauma or disease.

Since the introduction of the first commercial implantable stimulators in the late 1950s, there have been many devices approved for clinical use, resulting in a dramatic impact on the quality of life of millions of people around the world.

Five devices currently dominate neural stimulation from a clinical perspective: cochlear implants that stimulate the auditory nerve to treat profound hearing loss; spinal cord stimulation for treatment of severe chronic pain; vagal nerve stimulation to treat epilepsy and depression; deep-brain stimulation to alleviate the motor disorders associated with Parkinson's disease and essential tremor; and sacral root stimulation to provide bladder control in spinal cord injury. In addition to these five, there are many devices in development or an early stage of commercialization.

Recently, the field has experienced an exciting new phase of innovation generated by the National Institutes of Health's Stimulating Peripheral Activity to Relieve Conditions (SPARC) program, the Defense Advanced Research Projects Agency's ElectRx, SUBNETS, RAM, TNT and HAPTIX programs, and Galvani Bioelectronics bioelectronic therapies program. These initiatives call for significant multidisciplinary collaboration across the sciences, engineering, and clinical research.

Activities include the development of detailed anatomical and physiological maps of neural circuits associated with disease and the implementation of excitatory and/or inhibitory neuromodulation techniques for precise therapeutic stimulation. Peripheral nerves innervate and influence all organs of the body. Modulating neural activity via neural stimulation has the potential to treat a large number of diseases including inflammatory diseases, Type II diabetes, intestinal and bladder conditions, heart failure and hypertension.

Bioelectronics in Medicine provides this multidisciplinary sector with a high quality, rapid-to-publication journal. For publication enquiries please contact Commissioning Editor Sarah Jones (s.jones@futuremedicine.com). We hope you enjoy reading this inaugural issue, and look forward to your feedback.

\section{Financial \& competing interests disclosure}

The author has no relevant affiliations or financial involvement with any organization or entity with a financial interest in or financial conflict with the subject matter or materials discussed in the manuscript. This includes employment, consultancies, honoraria, stock ownership or options, expert testimony, grants or patents received or pending, or royalties.

No writing assistance was utilized in the production of this manuscript. 
